\title{
Estimation of Pulmonary Artery Catheter Length: A Retrospective Study
}

\author{
Naomi Ujino, Junko Nakahira*, Shoko Nakano, Toshiyuki Sawai and Toshiaki Minami \\ Department of Anesthesiology, Osaka Medical College, Japan
}

*Corresponding author: Junko Nakahira, Department of Anesthesiology, Osaka Medical College, Daigaku-machi, Takatsuki, Japan

\begin{abstract}
Background: Over-insertion of a pulmonary artery catheter (PAC) can cause serious complications, such as pulmonary embolism and pulmonary artery rupture. Careful management is essential.

Methods: This study investigated relationships between patient parameters and appropriate PAC insertion length. A function on the chest X-ray display was used to measure the curve. In Study 1, we investigated concordance using intraclass concordance coincidence (ICC) between recorded insertion length of the PAC and actual inserted length. We reviewed recorded insertion data from patients' anesthesia records and measured actual inserted length. In Study 2, we measured and estimated the appropriate insertion length using chest X-rays taken just after surgery. Multiple logistic regression was performed to investigate relationships between patient background parameters and appropriate insertion length.

Results: For Study 1, recorded insertion length was $41.2 \pm 2.7 \mathrm{~cm}$ and actual inserted length was $41.9 \pm 3.1 \mathrm{~cm}$. ICC $(2,1)$ was 0.867, which shows a high correlation. For Study 2, height and cardiac width on preoperative chest X-rays were significant factors determining appropriate PAC length. The multiple regression equation for appropriate PAC length ( $\mathrm{z}$ ) with height (x) and cardiac width $(\mathrm{y})$ was calculated as $\mathrm{z}(\mathrm{cm})=0.1 \mathrm{x}+0.8 \mathrm{y}+18.1$. Height and cardiac width were significant factors determining appropriate PAC insertion length.
\end{abstract}

Conclusions: We calculated an equation for the relationship between height and cardiac width to obtain the appropriate PAC insertion length.

Keywords: Pulmonary artery catheter; Right pulmonary artery; Chest X-ray

Abbreviations: ICC: intraclass concordance coincidence; PAC: pulmonary artery catheter; PCWP: pulmonary capillary wedge pressure.

\section{Background}

The pulmonary artery catheter (PAC) is used for many diagnostic applications, including measuring pulmonary artery pressure, cardiac output, and mixed venous oxygen saturation [1]. Over-insertion may cause serious complications, such as pulmonary embolism and pulmonary artery rupture. Careful management is essential. Approximately $90 \%$ of injuries occur at the medial and inferior branches of the right pulmonary artery, because a PAC inserted from the right internal jugular vein will most often enter the right pulmonary artery [2]. There are numerous reports of complications arising from pulmonary artery ruptures following PAC insertion. Such injury has a lower frequency (at 0.03-0.7\%) than infections, pulmonary embolism, pericarditis, and valvular vegetation as complications after cardiovascular surgery; however, it is a serious problem, with a mortality rate of $37 \%$ [3]. When inserting a PAC, the tip is recommended to be within $2 \mathrm{~cm}$ of the catheter silhouette on standard anteroposterior chest film [4]. PAC manufacturer instructions state that the best position for the tip is at the hilum of the lungs in the right pulmonary artery (see Additional file 1, p. 148). Position is determined by confirming pressure and waveform detected through the PAC tip. When waveform obtained from the tip of the PAC with the balloon inflated shows correct pulmonary capillary wedge pressure (PCWP), the tip must be in the 
pulmonary artery, and the balloon is deflated. Final confirmation of position is performed by chest X-ray postoperatively. The purpose of this study was to estimate appropriate PAC length from chest $\mathrm{X}$-rays and investigate relationships between the size of the body and heart and appropriate PAC length. A function on the chest $\mathrm{X}$-ray image display was used to measure the curve and evaluate PAC length. We hypothesized that the size of the heart or height and weight correlates with appropriate PAC length.

\section{Methods}

This study was a retrospective observational study of cardiovascular surgery patients in 2013. The study protocol was approved by the Ethics Committee of Osaka Medical College (Reference No. 1591Rin-47), which waived the requirement for informed consent because of the retrospective design of the study. Participants were patients in whom a PAC was inserted from the right internal jugular vein to the main or right pulmonary artery and who underwent surgery using cardiopulmonary bypass in the supine position. Emergency surgery and descending aorta replacement surgery were excluded. Induction of anesthesia was performed by two anesthesiologists. General anesthesia was induced by administration of propofol, midazolam, rocuronium, remifentanil, and sevoflurane. After endotracheal intubation, the right internal jugular vein was punctured at the cricoid cartilage level, the PAC sheath was inserted, then an 8Fr PAC (CCO/CEDV thermodilution catheter; 777HF8®; Edwards Lifesciences, Irvine, CA) was inserted through the sheath. A central venous catheter was inserted on the cranial side of the PAC insertion point. Intraclass concordance coincidence (ICC) was calculated in 20 cases in Study 1 to show concordance between recorded insertion length and actual inserted length. In Study 2, multiple regression analysis was performed to investigate relationships between appropriate insertion length and the parameters. Statistical analyses were performed using JMA9® (SAS Institute, Tokyo, Japan).

\section{Study 1}

A function on the X-ray imaging display was used to measure the curve. A chest X-ray was taken in the anteroposterior view immediately after surgery, with the patient in the supine position under general anesthesia. For Study 1, there were only 20 patients in whom the PAC was inserted from the cricoid cartilage, which was assumed to be at the level of the sixth cervical vertebra, over the study period. Anesthesiologists read the scale of the insertion length of the PAC just after surgery and recorded the data on anesthetic records as 'insertion length'. A chest X-ray was taken concurrently. The actual inserted length was measured with the display function using the postoperative chest X-ray. We confirmed the grade of concordance between recorded PAC insertion length collected from anesthesia records and actual inserted length. Using the curve measurement function, we measured PAC length from the middle of the sixth cervical vertebra to the tip of the PAC. The recorded insertion length was reviewed against anesthesia records. This

included $\sim 5.7 \mathrm{~cm}$ of the connector of the sheath; therefore, $5.7 \mathrm{~cm}$ was subtracted from reviewed data to obtain the correct length. To investigate concordance of the two parameters, we calculated the ICC. ICC is a descriptive statistic that can be used when quantitative measurements are made on units that are organized into groups. It describes how strongly units in the same group resemble each other. ICC $[1,2]$ means that each subject is measured by each rater, and raters are considered representative of a larger population of similar raters. Reliability is calculated from a single measurement. It was determined that $>0.7$ ICC $[1,2]$ meant high concordance between recorded insertion length and actual PAC length.

\section{Study 2}

Patient backgrounds were reviewed from patient records and chest X-rays taken preoperatively. Cardiac width was the transverse diameter of the heart in the posterior-anterior view in a standing position as the widest diameter of the cardiac silhouette on each side of a central perpendicular line. Thoracic width was the widest diameter of the chest in the posterior-anterior view taken at a distance between the internal surface of the ribs on the right and left sides, superior to the costal attachment of the diaphragm [5]. Cardiothoracic ratio was calculated as the ratio of cardiac width divided by thoracic width. At our institute, over the period in which Study 2 was conducted, there were 395 patients who underwent cardiovascular surgery, including pediatric cardiovascular surgery and vascular surgery, amongst others. There were 149 cases of adult surgery, not including abdominal aorta replacement and stenting. Elective surgery that was performed through median sternotomy was included. Of these 149 cases, 121 met inclusion criteria for Study 2. Appropriate insertion lengths of the PAC were estimated and measured in these 121 patients. The curve measuring function on the display was used with a chest X-ray taken just after cardiac surgery in the operating room. Actual inserted length was measured from the sixth cervical vertebra to the tip of the PAC. In cases where the PAC did not reach the hilum, we estimated the course of the PAC and added the distance from the inserted length to the hilum.

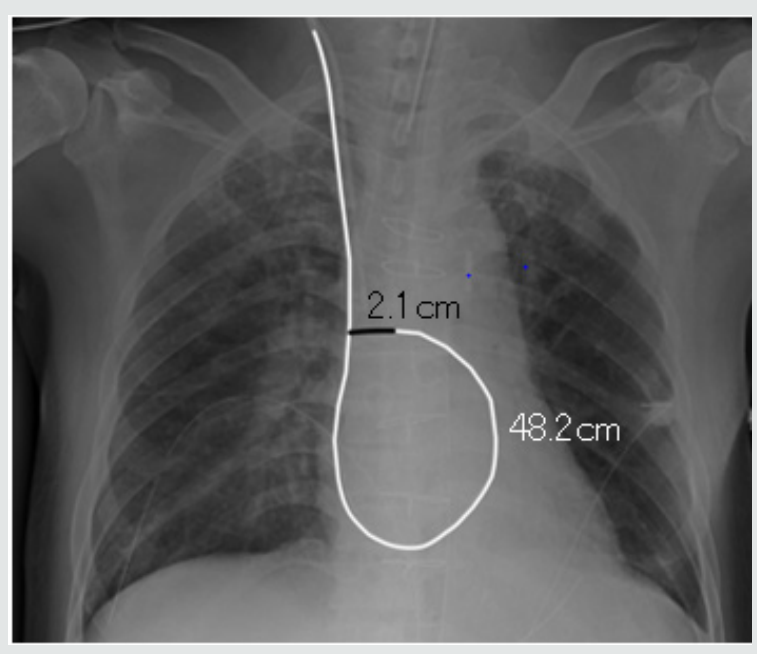

Figure 1 
We determined that the appropriate position of the PAC tip was the intersection of the PAC in the superior vena cava and the PAC tip in the right pulmonary artery. (Figure 1) shows how the inserted length was measured. The white line shows the inserted PAC and the black line shows estimated distance from the tip to the hilum. The actual inserted length was $48.2 \mathrm{~cm}$ and the estimated added length was $2.1 \mathrm{~cm}$. The appropriate insertion length was therefore calculated as $50.3 \mathrm{~cm}$. When the PAC exceeded the hilum in the X-ray, the appropriate length was determined as the length from the sixth cervical vertebra to the hilum of the lung.

\section{Results}

Table 1: Patient backgrounds and measurements in Study 2 $(n=121)$.

\begin{tabular}{|l|c|}
\hline Variables & \\
\hline Age (years) & $71 \pm 10$ \\
\hline Male/female & $83 / 38$ \\
\hline Height (cm) & $160.7 \pm 8.6$ \\
\hline Weight (kg) & $58.0 \pm 11.6$ \\
\hline Cardiac width (cm) & $15.1 \pm 1.9$ \\
\hline Thoracic width (cm) & $28.6 \pm 2.5$ \\
\hline Cardiothoracic ratio (\%) & $52.9 \pm 7.0$ \\
\hline Actual inserted length of PAC (cm) & $42.9 \pm 4.1$ \\
\hline $\begin{array}{l}\text { Appropriate insertion length of } \\
\text { PAC (cm) }\end{array}$ & $46.6 \pm 3.5$ \\
\hline
\end{tabular}

PAC: pulmonary artery catheter

Table 2: Results of multiple logistic regression in Study 2.

\begin{tabular}{|l|c|}
\hline Variables & \\
\hline Age (years) & $71 \pm 10$ \\
\hline Male/female & $83 / 38$ \\
\hline Height (cm) & $160.7 \pm 8.6$ \\
\hline Weight (kg) & $58.0 \pm 11.6$ \\
\hline Cardiac width (cm) & $15.1 \pm 1.9$ \\
\hline Thoracic width (cm) & $28.6 \pm 2.5$ \\
\hline Cardiothoracic ratio (\%) & $52.9 \pm 7.0$ \\
\hline Actual inserted length of PAC (cm) & $42.9 \pm 4.1$ \\
\hline $\begin{array}{l}\text { Appropriate insertion length of } \\
\text { PAC (cm) }\end{array}$ & $46.6 \pm 3.5$ \\
\hline
\end{tabular}

$\beta$ : standardized partial regression coefficient: $r$ : correlation coefficient; ${ }^{* *} \mathrm{p}<0.01$

For Study 1, recorded insertion length was $41.2 \pm 2.7 \mathrm{~cm}$ and actual inserted length measured using chest X-ray was $41.9 \pm 3.1 \mathrm{~cm}$. ICC $(2,1)$ was 0.867 , which shows a high correlation. For Study 2 , (Table 1) shows patient backgrounds and measurements. There were nine cases in which the PAC tip was inserted exceeding the right hilum. Height and cardiac width on preoperative chest X-rays were significant factors in determining appropriate inserted PAC length $(\mathrm{P}<0.05)$ (Table 2). The multiple regression equation for appropriate PAC length $(\mathrm{z})$ with height $(\mathrm{x})$ and cardiac width $(\mathrm{y})$ was calculated as $\mathrm{z}(\mathrm{cm})=0.1 \mathrm{x}+0.8 \mathrm{y}+18.1$.

\section{Discussion}

We hypothesized that PAC length is affected by the size of the body and heart. Our results aligned with values previously reported, despite differences in pulmonary artery pressure, cardiac diseases, and loop formation depending on the catheter. When using average values for Japanese people with normal blood pressure (male height: $168.6 \mathrm{~cm}$; female height: $156.1 \mathrm{~cm}$; cardiac length for both males and females: $13.8 \mathrm{~cm}$ ) in our equation, the appropriate insertion PAC length was calculated as $46.0 \mathrm{~cm}$ for men and $44.8 \mathrm{~cm}$ for women. Participants were patients with cardiac diseases requiring surgery, and cardiac width was, on average, $15.1 \mathrm{~cm}$, which is greater than the Japanese average. This may explain why the appropriate insertion length in our study was greater than average values. PAC insertion length for obtaining PCWP is commonly determined as $45-55 \mathrm{~cm}$ [4]. Researchers in Japan and India, where average height is shorter, reported the appropriate insertion length as $\sim 40 \mathrm{~cm}$, with length tending to be greater in valvular disease patients $[6,7]$. Studies have reported that PAC length from the right internal jugular vein to the PAC tip for obtaining PCWP is $\sim 44 \pm 6 \mathrm{~cm}$ and $42-48 \mathrm{~cm}$, and no correlation between height and appropriate PAC length was noted by the Japanese researchers $[8,9]$. Another study suggests that PAC length for obtaining PCWP is commonly $<50 \mathrm{~cm}$. When the inserted PAC length is $>50 \mathrm{~cm}$, PAC insertion should be retried, or the position assessed using chest X-ray or fluoroscopy as there may be sagging or loop formation [9]. According to one manufacturer (Edwards Life Sciences), the recommended PAC tip position is at the hilum. At this position, pressure waveform shows PCWP when the balloon is inflated. At this stage, the balloon should be deflated and pulled back $\sim 2-3 \mathrm{~cm}$ slowly to avoid sagging and loop formation in the right atrium and ventricle. Pulmonary artery rupture by PAC occurs rarely but is a critical complication. Pulmonary hypertension, anticoagulation therapy, old age, low body temperature, displacement of the PAC tip, balloon overinflation, or heart surgery are reported risk factors for rupture [3]. Cardiovascular surgery includes multiple factors that can cause rupture, therefore, careful observation of PAC insertion length is needed. Although there is a lack of data on the effectiveness of PAC in cardiac surgery [10], parameters taken from the PAC are relied on and it remains a standard procedure in many practices.

\section{Conclusion}

Height and cardiac width were significant factors determining the appropriate PAC insertion length. We calculated an equation for the relationship between height and cardiac width to obtain the appropriate PAC insertion length. 


\section{Ethics approval and Consent to Participate}

The study protocol was approved by the Ethics Committee of Osaka Medical College (Reference No. 1591Rin-47), which waived the requirement for informed consent because of the retrospective design of the study.

\section{References}

1. American Society of Anesthesiologists Task Force on Pulmonary Artery Catheterization (2003) Practice guidelines for pulmonary artery catheterization: an updated report by the American Society of Anesthesiologists Task Force on Pulmonary Artery Catheterization. Anesthesiology 99: 988-1014.

2. Urschel JD, Myerowitz PD (1993) Catheter-induced pulmonary artery rupture in the setting of cardiopulmonary bypass. Ann Thorac Surg 56: $585 \mathrm{M} 9$.

3. Kearney TJ, Shabot MM (1995) Pulmonary artery rupture associated with the Swan-Ganz catheter. Chest. 108(5): 1349-1352.

4. Miller RD (2014) Chapter 45: Cardiovascular monitoring. In: Miller's Anesthesia. 8th ed. Philadelphia: Elsevier Saunders, pp. 1370-1371.
5. Inoue K, Yoshii K, Ito H (1999) Effect of aging on cardiothoracic ratio in women: a longitudinal study. Gerontology 45: 53-58.

6. Ishida K (1996) Use experience of Swan-Ganz catheter (in Japanese). Kokura Kinen Hospital Journal of Medicine 29: 44-48.

7. Tempe DK, Gandhi A, Datt V, Gupta M, Tomar AS, et al. (2006) Length of insertion for pulmonary artery catheters to locate different cardiac chambers in patients undergoing cardiac surgery. Br J Anaesth 97(2): 147-149.

8. Kaieda R, Inoue S, Waki M (1984) The length of the pulmonary artery catheter though the internal jugular vein (in Japanese). Rinsyomasui (Journal of Clinical Anesthesia) 8: 440-442.

9. Suzuki T, Kinefuchi Y, Miura M, Takiguchi M, Yamasaki Y, et al. (1990) Swan-Ganz catheters: distances from the site of insertion to the right ventricle and pulmonary artery, and forward displacement of catheter tips (in Japanese with English abstract). Circulation Control 11: 485-488.

10. Schwann NM, Hillel Z, Hoeft A, Barash P, Möhnle P et al. (2011) Lack of effectiveness of the pulmonary artery catheter in cardiac surgery. Anesth Analg 113: 994-1002.

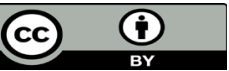

This work is licensed under Creative Commons Attribution 4.0 License

To Submit Your Article Click Here:

Submit Article

DOI: $10.32474 /$ GJAPM.2019.01.000108

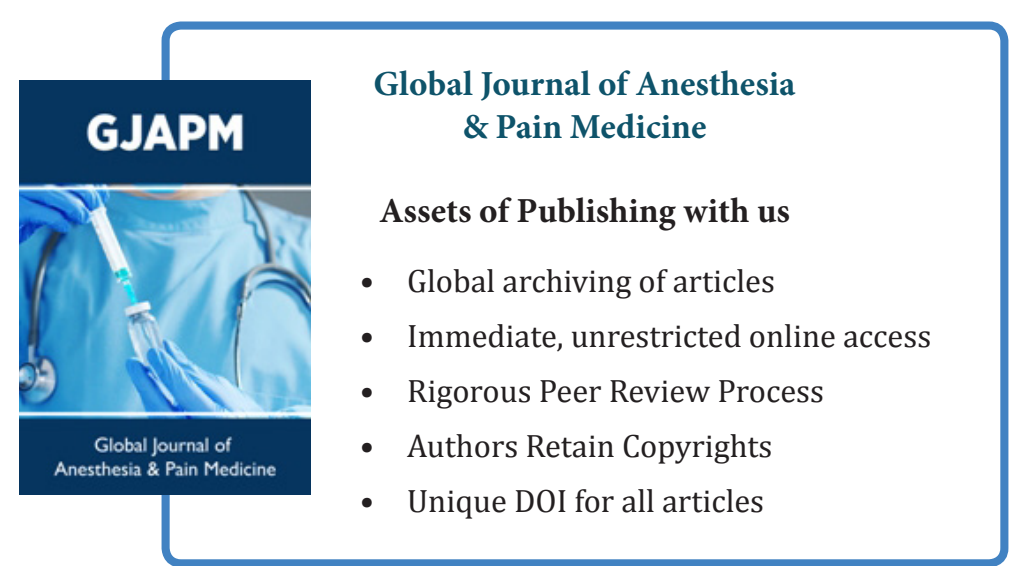

